\title{
Creencias epistemológicas de estudiantes de pedagogía: validación del cuestionario y análisis de diferencias
}

\author{
MAYRA CUÉLLAR-FAJARDO* \\ Pontificia Universidad Católica del Perú \\ FRANCESC MARTÍNEZ-OLMO** \\ Universitat de Barcelona - España \\ Recibido el 12-07-2016; primera evaluación el 26-07-2017; segunda evaluación \\ el 26-07-2017; tercera evaluación el 01-08-2017; aceptado el 15-08-2017
}

\section{RESUMEN}

El presente estudio describe las creencias epistemológicas de los estudiantes de pedagogía de la Universidad de Barcelona (España) y analiza diferencias en sus creencias debido al momento de formación, sexo y edad. Para ello se estimaron las propiedades psicométricas (validez de constructo y consistencia interna) del Cuestionario de Creencias Epistemológicas de Schommer (1990). Participaron 229 estudiantes. Los resultados muestran que la fiabilidad del instrumento es moderada $(\alpha=.65)$ y la estructura factorial hallada valida el modelo epistemológico propuesto por Schommer. Asimismo, los resultados indican que las creencias de los estudiantes de último año son más sofisticadas respecto a la habilidad para aprender, estructura y

\footnotetext{
Máster en Investigación y Cambio Educativo por la Universitat de Barcelona, España. Psicóloga social egresada de la Pontificia Universidad Católica del Perú (PUCP). Ha colaborado en la docencia de la asignatura de posgrado "Evaluación Educativa" de la Universitat de Barcelona. Actualmente brinda soporte en el proceso de acreditación internacional de la calidad educativa de la Especialidad de Sociología (Facultad de Ciencias Sociales) de la Pontificia Universidad Católica del Perú (PUCP). Contacto: mayra.cuellar@pucp.pe

** Pedagogo. Profesor doctor de la Universitat de Barcelona y especialista en Métodos de Investigación y Diagnóstico en Educación. Miembro del grupo de investigación consolidado en enseńanza y aprendizaje virtual (GREAV) y del grupo de innovación docente MideMe. Secretario del Seminario Permanente de Orientación Profesional (SEPEROP). Codirector de la Revista de Innovación e Investigación en Educación (REIRE) y director del máster de Profundización en el primer ciclo de Educación Infantil. Contacto: fmartinezo@ub.edu
} 
certeza del conocimiento, en comparación a los estudiantes de primer año. También se encontraron diferencias debido al sexo, pero no debido a la edad.

Palabras clave: creencias epistemológicas, educación superior, propiedades psicométricas (validez y fiabilidad)

Epistemological beliefs of students of Pedagogy: validation of the questionnaire and analysis of differences

\section{Abstract}

This study describes epistemological beliefs from students in the Degree of Pedagogy at the University of Barcelona (Spain), and analyzes differences in these beliefs according to their year of study, gender, and age. To do so, the psychometric properties (construct validity and internal consistency) of Schommer Epistemological Questionnaire (1990) were estimated. 229 students participated. Results show that the reliability of the instrument is moderate $(\mathrm{a}=.653)$, and the factorial structure revealed validates the epistemological model proposed by Schommer. Furthermore, the results indicate that final year student beliefs are more sophisticated than first year student beliefs in respect to learning ability and to the structure and certainty of knowledge. Differences were also found owning to the student's gender, but not to age.

Keywords: epistemological beliefs, higher education, psychometric properties (validity and reliability)

Crenças epistemológicas dos estudantes de pedagogia: validação do questionário e análise das diferenças

\section{Resumo}

Os objetivos deste estudo são descrever as crenças epistemológicas dos estudantes de pedagogia da Universidade de Barcelona (Espanha), e analisar diferenças em suas crenças, tendo em conta a formação em estudos de ensino superior (primeiro e no último ano), sexo e idade. Para fazê-lo, se fez uma estimação das propriedades psicométricas (validade de construto e consistência interna) do Questionário de Crenças Epistemológicas (Schommer, 1990), com a participação de 229 estudantes. Os resultados mostram que a confiabilidade do instrumento é moderada ( $\alpha$ $=0,65)$ e que a estrutura de fatores valida o modelo epistemológico de Schommer. Os resultados também indicam que as crenças dos estudantes do último ano são mais sofisticadas sobre a capacidade de aprender e a estrutura e a certeza do conhecimento, isto em comparação com os estudantes do primeiro ano. Houve também diferenças devido ao sexo, mas náo por causa da idade.

Palavras-chave: crenças epistemológicas, ensino superior, propriedades psicométricas (validade e confiabilidade) 


\section{INTRODUCCIÓN}

Desde finales de la segunda mitad del siglo XX se han venido realizando, cada vez con mayor profusión, investigaciones que buscan conocer el aprendizaje desde la perspectiva del estudiante y cómo este adquiere, procesa y maneja la información presentada por los docentes en los contextos educativos. Estas tendencias en la investigación coinciden con el paradigma educativo actualmente en boga, que concibe el aprendizaje como construcción de significado, por lo que el foco de atención dentro del proceso formativo está cada vez más siendo puesto en el estudiante y en cómo este construye el conocimiento a partir de sus experiencias previas, interpreta, transforma y da sentido a lo que aprende (De Juanas \& Beltrán 2012).

En tiempos recientes, varios investigadores han mostrado especial interés para estudiar las creencias o teorías que las personas sostienen acerca del conocimiento y el aprendizaje, lo cual les ha permitido reconocer cómo estas creencias epistemológicas o epistemologias personales se relacionan directa o indirectamente con el aprendizaje académico (Hofer \& Pintrich, 2002; Wang, Zhang, Zhang \& Hou, 2013). El contenido de las creencias epistemológicas es sobre dos aspectos fundamentales: la naturaleza del conocimiento y el proceso de adquisición del conocimiento (Hofer, 2004; Pecharromán \& Pozo, 2010). Específicamente, dichas creencias reflejan los puntos de vista personales acerca de qué es el conocimiento, cómo se estructura, cuál es el grado de certeza o de verdad del conocimiento, cómo se adquiere y de dónde proviene el conocimiento (Coşkun \& Grainger, 2014).

Las creencias epistemológicas son construcciones socioculturales que reflejan el contexto de aprendizaje y su tipo de pensamiento subyacente (Buelh \& Alexander, 2006; Schommer-Aikins, Beuchat-Reichardt \& HernándezPina, 2012), por lo que las ideas acerca del aprendizaje y la enseñanza pueden ser ideas periféricas precursoras del desarrollo de ideas centrales acerca de la epistemología (Hofer \& Pintrich, 1997).

Desde que William Perry (1968) teorizó por vez primera el desarrollo epistemológico de los estudiantes, varios investigadores continuaron su línea de investigación y han reconocido la influencia de estas creencias en el aprendizaje y la enseñanza (Topcu, 2013). Actualmente la concepción multidimensional de las creencias epistemológicas propuesta por Marlene Schommer (1990) es la que goza de gran influencia en el campo, al afirmar que la epistemología personal es un sistema de creencias más o menos independientes. Es decir, una persona puede demostrar madurez (sofisticación) en algunas creencias e inmadurez (ingenuidad) en otras (Schommer-Aikins \& Easter, 2008). 
La evolución o desarrollo de las creencias se apreciaría justamente en el paso de los sujetos de creencias ingenuas a creencias sofisticadas (Müller, Rebmann \& Liebsch, 2008).

Schommer (Duell \& Schommer-Aikins, 2001) propuso un modelo epistemológico compuesto de cinco creencias (ver tabla 1) que tratan acerca de la estructura del conocimiento (conocimiento simple), estabilidad del conocimiento (conocimiento cierto), fuente del conocimiento (autoridad omnisciente), habilidad para aprender (habilidad fija) y rapidez en el aprendizaje (aprendizaje rápido). Además, elaboró un cuestionario para evaluar esas cinco creencias; sin embargo, después de poner a prueba su modelo en diferentes oportunidades, no logró validar empíricamente la dimensión autoridad omnisciente.

Tabla 1. Creencias epistemológicas según el modelo teórico de Schommer

\begin{tabular}{lll}
\hline \multicolumn{1}{c}{ Creencia } & \multicolumn{1}{c}{ No sofisticada } & \multicolumn{1}{c}{ Sofisticada } \\
\hline $\begin{array}{l}\text { Conocimiento } \\
\text { simple }\end{array}$ & $\begin{array}{l}\text { El conocimiento es simple, } \\
\text { está formado de datos aislados }\end{array}$ & $\begin{array}{l}\text { El conocimiento es complejo, es } \\
\text { una red de conceptos entrelazados }\end{array}$ \\
$\begin{array}{l}\text { Conocimiento } \\
\text { cierto }\end{array}$ & $\begin{array}{l}\text { El conocimiento es cierto, no } \\
\text { cambia }\end{array}$ & $\begin{array}{l}\text { El conocimiento es algo provisio- } \\
\text { nal y cambiante }\end{array}$ \\
$\begin{array}{l}\text { Autoridad } \\
\text { omnisciente }\end{array}$ & $\begin{array}{l}\text { El conocimiento se transmite } \\
\text { desde la autoridad }\end{array}$ & $\begin{array}{l}\text { El conocimiento proviene de } \\
\text { la razón, es una construcción } \\
\text { personal }\end{array}$ \\
Habilidad fija & $\begin{array}{l}\text { La habilidad para aprender es } \\
\text { innata y no cambia }\end{array}$ & $\begin{array}{l}\text { La habilidad para aprender puede } \\
\text { mejorarse }\end{array}$ \\
$\begin{array}{l}\text { Aprendizaje } \\
\text { rápido }\end{array}$ & $\begin{array}{l}\text { El aprendizaje ocurre desde } \\
\text { un primer momento o no }\end{array}$ & El aprendizaje es gradual \\
& ocurre & \\
\hline
\end{tabular}

Fuente: Elaboración propia con información proveniente de Duell y Schommer (2001).

No se debe asumir que una persona es madura en sus creencias epistemológicas solo porque demuestra madurez en una o algunas de sus creencias (Schommer-Aikins \& Duell, 2013). Además, los extremismos en las creencias pueden ser contraproducentes. Por ejemplo, considerar el conocimiento como totalmente inestable podría ocasionar en alguien dificultades para asumir posturas personales; en contraste, considerar el conocimiento como completamente invariable podría conducir a la rigidez cognitiva (Schommer-Aikins, 2004). No obstante, el rol benéfico que cumplen las creencias epistemológicas que tienden a la sofisticación ha sido validado muchas veces en estudios realizados durante las últimas dos décadas (Trautwein \& Lüdtke, 2007). 
En general, creencias maduras se asocian a orientaciones constructivistas de la enseñanza y el aprendizaje (García \& Sebastián, 2011; Martínez, Montero $\&$ Pedrosa, 2009) y a desempeños sobresalientes en diferentes tareas (Mason \& Boscolo, 2004; Schommer-Aikins, 2004); a diferencia de las creencias ingenuas, que comúnmente acompañan un nivel bajo de aprendizaje, donde predomina la memorización (Schommer-Aikins et al., 2012).

\section{CREenCias ePISTEMOlógiCAS, EDAD, EDUCACión Y DifERENCIAS DE SEXo}

Parece cobrar más fuerza la idea de que la maduración de las creencias no ocurre por acción de la edad, sino más bien por la exposición a experiencias educativas (Hofer, 2001; Hofer \& Pintrich, 1997; Schommer, 1993, 1998). Eso también depende de si los contextos educativos brindan oportunidades para que las preconcepciones que los estudiantes traen consigo a la formación se activen y confronten sistemáticamente mediante la presentación de nuevos conceptos admisibles y útiles, que los ayuden a comprender de una mejor manera la realidad (Gill, Ashton \& Algina, 2004), y de si la instrucción se enfoca en la discusión y el análisis crítico de problemas complejos que impliquen la integración de información (Cho \& Lankford, 2011; Jehng, Johnson \& Anderson, 1993; Mohamed \& El-Habbal, 2013).

Respecto a las diferencias de sexo y su relación con las creencias epistemológicas, son pocas las investigaciones que brindan resultados concluyentes. Algunos resultados empíricos coinciden en afirmar que las mujeres tienden a creer en menor medida tanto en un aprendizaje rápido como en la habilidad innata para aprender (De Juanas \& Beltrán, 2012; Schommer, 1993). Los estudios en hombres evidencian resultados contrapuestos: Hofer (2000) halló que los hombres tienen más probabilidades de concebir el conocimiento como cierto y proveniente de la autoridad, mientras que un estudio realizado con una muestra de estudiantes turcos de escuela básica reveló que los varones mostraban creencias más inciertas acerca del conocimiento en comparación a las mujeres (Ozkal, Tekkaya, Sungur, Cakiroglu \& Cakiroglu, 2011).

\section{OBJETIVOS, HIPÓTESIS Y MÉTODO DE LA INVESTIGACIÓN}

Los objetivos del estudio fueron describir las creencias epistemológicas de los estudiantes de pedagogía de la Universidad de Barcelona y analizar si existen diferencias en sus creencias debido al momento de formación en los estudios (primer y último año), sexo y edad. Para ello se estimó la validez y consistencia interna del Cuestionario de Creencias Epistemológicas de Schommer (1990). 
La investigación se enfocó en estudiantes de pedagogía ya que por la idiosincrasia de su profesión requieren destrezas cognitivas para la comprensión del proceso de conocimiento y aprendizaje, en coherencia con los nuevos modelos de reforma educativa vigentes que fomentan la búsqueda de significado, el ejercicio de la razón, la flexibilidad conceptual y el aprender a aprender. Lo esperado sería que sus creencias epistemológicas coincidan con los principios pedagógicos actuales. Asimismo, el conocimiento de las creencias epistemológicas de los estudiantes puede aportar información valiosa para el diseńo y desarrollo de la docencia universitaria (De Juanas \& Beltrán, 2012) y para la promoción de contextos educativos que favorezcan la maduración de las epistemologías personales (Han \& Jeong, 2014; Kember, Hong, Yau \& Ho, 2014). En relación a los objetivos descritos se plantearon las siguientes hipótesis:

H1: Las creencias epistemológicas de las y los estudiantes de pedagogía de la Universidad de Barcelona se diferencian según el momento de formación en sus estudios. Es decir, las y los estudiantes de último año tendrán creencias más sofisticadas que las y los estudiantes del primer año.

H2: Las creencias epistemológicas de las y los estudiantes de pedagogía de la Universidad de Barcelona se diferencian según el sexo.

H3: Existe una relación entre la edad y las creencias epistemológicas. A mayor edad de las y los estudiantes, más sofisticadas serán sus creencias.

La investigación utilizó un método cuantitativo y el diseño fue no experimental de tipo transversal, descriptivo y correlacional. Es transversal debido a que la recolección de todos los datos se realizó en un mismo momento; descriptivo, dado que se describen las creencias epistemológicas en una población determinada y se realizan comparaciones y correlaciones atendiendo a las variables de interés (edad, año de estudios y sexo). El estudio se enmarcó además dentro de los estudios por encuesta, dado que se utilizó un cuestionario cerrado para la recogida de información.

\subsection{Participantes}

Se llevó a cabo un muestreo no probabilístico de tipo incidental. Se aplicó el instrumento a los estudiantes matriculados en dos asignaturas que componen el plan de estudios del grado de pedagogía. Una de las asignaturas se imparte 
en el bloque de formación básica en pedagogía correspondiente a los estudios de primer año. La otra asignatura se encuentra en el bloque de formación obligatoria que corresponde al último año (cuarto) de los estudios.

La muestra integró 229 participantes, todos estudiantes del curso académico 2014-2015. 155 estudiantes correspondían al primer ańo (67,7\%) y 74 estudiantes al cuarto año (32,3\%). Sobre el total de participantes, 204 fueron mujeres $(89,1 \%)$, lo cual se debe a que más del $80 \%$ de la población matriculada en el grado de pedagogía es del sexo femenino. Las edades fluctuaron entre los 18 y 47 ańos, aunque el 90,4\% de los participantes de la muestra tenía una edad que se ubicaba dentro del rango de 18-24 ańos.

\subsection{Instrumento}

La recogida de datos se realizó con el Schommer Epistemological Questionnaire (SEQ) que cuenta con un total de 63 ítems agrupados en 12 subescalas que conforman las 5 dimensiones de las creencias epistemológicas propuestas por Schommer (1990) que son: Conocimiento simple, Conocimiento cierto, Autoridad omnisciente, Habilidad fija y Aprendizaje rápido. La respuesta a cada ítem se estimó de acuerdo a una escala Likert de cinco valores donde 1 es totalmente en desacuerdo y 5 totalmente de acuerdo. Anteriores aplicaciones del instrumento comprobaron la validez de constructo solo para 4 de sus dimensiones (Autoridad omnisciente no ha logrado ser validada empíricamente). En esta investigación se utilizó la traducción del SEQ (Cuestionario de Creencias Epistemológicas -CCE-) elaborada por Di Matteo (2007), la cual se cotejó con el cuestionario de Schommer en su versión original anglosajona. Aproximadamente el $50 \%$ de los ítems fueron parafraseados y ajustados terminológicamente a fin de hacerlos más comprensibles a los participantes procurando una traducción semántica del idioma original del cuestionario. Por ejemplo, uno de los ítems en inglés dice "If I find the time to re-read a textbook chapter, I get a lot more out of it the second time". Dicho enunciado fue traducido por Di Matteo (2007) a "Cuando tengo tiempo de releer un texto obtengo mucho mayor provecho", que en el presente estudio fue ajustado a "Si tengo tiempo para releer un capítulo de un texto, obtengo mayor provecho esa segunda vez".

\subsection{Procedimiento}

El proceso de recogida de información se realizó en las semanas previas al término de las clases del segundo semestre del curso académico 2014-2015. 
Para el levantamiento de información se solicitó el consentimiento del jefe de estudios del grado de pedagogía, de los docentes y de los alumnos. La participación de los estudiantes fue voluntaria por lo que podían desistir en cualquier momento de completar el cuestionario si así lo quisieran. Se aseguró la confidencialidad de la información recabada. No se solicitaron los datos personales de los participantes, salvo los datos de sexo y edad.

\subsection{Análisis estadísticos}

Para efectuar los análisis cuantitativos de la información recabada se registraron las respuestas de los participantes en una hoja de cálculo del programa MS Excel. Luego, se importaron esos datos al programa estadístico SPSS versión 20 y se procedió a realizar diferentes tipos de análisis: análisis de fiabilidad y de reducción de factores (para determinar la consistencia interna del instrumento y revalidar su estructura), análisis descriptivos de la muestra y de las dimensiones del instrumento, pruebas de ajuste a la normalidad, análisis de contraste de medias no paramétricos y análisis de correlación.

\section{RESULTADOS: VALIDACIÓN DE CUESTIONARIO Y ANÁLISIS DE DIFERENCIAS}

\subsection{Análisis de fiabilidad del CCE}

El coeficiente de fiabilidad se obtuvo mediante el alfa de Cronbach, cuya puntuación obtenida $(\alpha=.65)$ indicó para el cuestionario una consistencia interna moderada y aceptable, considerando que el análisis realizado fue de tipo exploratorio. Al mismo tiempo, dicho valor alfa es tomado con precaución puesto que este depende de la unidimensionalidad del cuestionario que, aunque mide el constructo de creencias epistemológicas, lo realiza a nivel multidimensional.

\subsection{Análisis factorial del CCE}

A fin de validar las dimensiones teóricas propuestas por la autora (Schommer, 1990), se optó por efectuar un análisis factorial, pero antes se comprobó la pertinencia de dicho análisis examinando los valores obtenidos por la medida de adecuación muestral de Kaiser-Meyer Olkin $(\mathrm{KMO}=.63)$ y la prueba de esfericidad de Bartlet $\left({ }^{2}\right.$ aproximado $\left.=275.842, p=.00\right)$. Ambos métodos fueron consistentes al revelar que sí es apropiado efectuar un análisis de factores.

Para realizar el análisis factorial se trataron las 12 subescalas como variables, tal como lo efectúa la autora del cuestionario (Schommer, 1990, 1993). 
Mediante el método de extracción de componentes principales se obtuvieron 5 factores que explican el $60.05 \%$ de la varianza (ver tabla 2 ).

Tabla 2. Varianza total explicada por el análisis factorial del CCE

\begin{tabular}{|c|c|c|c|c|c|c|c|c|c|}
\hline \multirow{2}{*}{$\begin{array}{c}\text { Compo- } \\
\text { nentes }\end{array}$} & \multicolumn{3}{|c|}{ Autovalores Iniciales } & \multicolumn{3}{|c|}{$\begin{array}{c}\text { Sumas de las saturacio- } \\
\text { nes al cuadrado de la } \\
\text { extracción }\end{array}$} & \multicolumn{3}{|c|}{$\begin{array}{l}\text { Sumas de las saturacio- } \\
\text { nes al cuadrado de la } \\
\text { rotación }\end{array}$} \\
\hline & Total & $\begin{array}{c}\% \text { de la } \\
\text { varianza }\end{array}$ & $\begin{array}{c}\% \\
\text { acumul. }\end{array}$ & Total & $\begin{array}{c}\% \text { de la } \\
\text { varianza }\end{array}$ & $\begin{array}{c}\% \\
\text { acumul. }\end{array}$ & Total & $\begin{array}{l}\% \text { de la } \\
\text { varianza }\end{array}$ & $\begin{array}{c}\% \\
\text { acumul. }\end{array}$ \\
\hline 1 & 2.290 & 19.082 & 19.082 & 2.290 & 19.082 & 19.082 & 1.597 & 13.307 & 13.307 \\
\hline 2 & 1.643 & 13.696 & 32.778 & 1.643 & 13.696 & 32.778 & 1.563 & 13.028 & 26.334 \\
\hline 3 & 1.169 & 9.742 & 42.520 & 1.169 & 9.742 & 42.520 & 1.501 & 12.510 & 38.845 \\
\hline 4 & 1.100 & 9.163 & 51.683 & 1.100 & 9.163 & 51.683 & 1.285 & 10.708 & 49.553 \\
\hline 5 & 1.004 & 8.370 & 60.053 & 1.004 & 8.370 & 60.053 & 1.260 & 10.500 & 60.053 \\
\hline 6 & .955 & 7.960 & 68.013 & & & & & & \\
\hline 7 & .815 & 6.790 & 74.803 & & & & & & \\
\hline 8 & .736 & 6.130 & 80.933 & & & & & & \\
\hline 9 & .665 & 5.543 & 86.475 & & & & & & \\
\hline 10 & .609 & 5.077 & 91.552 & & & & & & \\
\hline 11 & .531 & 4.425 & 95.977 & & & & & & \\
\hline 12 & .483 & 4.023 & 100.000 & & & & & & \\
\hline
\end{tabular}

Fuente: Elaboración propia con información proveniente de los análisis estadísticos efectuados en el presente estudio.

La rotación Varimax fue seleccionada para interpretar mejor los factores obtenidos y aplicar el mismo tipo de rotación que Schommer utilizó en sus estudios (1990, 1993, 1998). A continuación, en la tabla 3, se muestran las saturaciones de la estructura factorial resultante que validan las 5 dimensiones teóricas propuestas por Schommer (1990). Es importante resaltar que, en el caso de las subescalas "Se debe evitar integrar información” y "El aprendizaje es rápido", se tomó en cuenta para su agrupación en un factor no las saturaciones más altas sino aquellas saturaciones que, en su vinculación, componen y explican de una mejor manera la estructura factorial según la propuesta teórica de Schommer (1990). Esto fue factible ya que las saturaciones obtenidas para cada una de estas dos subescalas fueron en todos los casos muy potentes. 
Tabla 3. Matriz de la estructura factorial rotada del CCE

\begin{tabular}{lccccc}
\hline \multicolumn{1}{c}{ Subescalas } & Factor & Factor & & Factor & Factor \\
& 1 & 2 & & 4 & 5 \\
\hline La habilidad para aprender es innata & .700 & & & & \\
Se debe evitar la ambigüedad & .632 & & & & \\
Concentrarse es una pérdida de tiempo & .601 & & -.409 & & \\
No se debe criticar a la autoridad & & .794 & & & \\
El aprendizaje es rápido & & .515 & & .450 & \\
Se debe evitar integrar información & .456 & .512 & & & \\
Se debe buscar respuestas simples & & & .787 & & \\
El conocimiento es cierto & & & .725 & & \\
El conocimiento proviene de la autoridad & & .351 & & -.723 & \\
Aprendizaje ocurre en el primer intento & & .311 & & .665 & \\
No se puede aprender a aprender & & & & & .764 \\
Éxito no relacionado con el esfuerzo & & & & & .724 \\
\hline
\end{tabular}

Método de extracción: Análisis de componentes principales. Método de rotación: Normalización Varimax con Kaiser.

Fuente: Elaboración propia con información proveniente de los análisis estadísticos efectuados en el presente estudio.

A continuación se procede a describir las subescalas que se decidió agrupar en cada factor:

Factor 1 Conocimiento simple: Este factor refiere a la estructura del conocimiento. Incluye las subescalas "La habilidad para aprender es innata", "Se debe evitar la ambigüedad", "Concentrarse es una pérdida de tiempo" y "Se debe evitar integrar información”. Se denominó a este factor conocimiento simple puesto que saturan en él dos de las subescalas que en anteriores investigaciones de Schommer (1990, 1993, 1998) se han agrupado siempre en este factor ("Se debe evitar la ambigüedad" y "Se debe evitar integrar información”).

Factor 2 Autoridad omnisciente: Este factor remite a la fuente del conocimiento. En los anteriores estudios efectuados por Schommer (1990, 1992, 1993), las subescalas "No se debe criticar a la autoridad" y "El Conocimiento depende de la autoridad" han saturado en diferentes factores, a pesar de que el contenido de los ítems refiere a una sola dimensión teórica. En el presente 
estudio, no obstante, se comprueba, por primera vez en un estudio empírico, la correlación de ambas subescalas dentro de un mismo factor.

Factor 3 Conocimiento cierto: Este factor refiere a la existencia de un conocimiento único, verdadero e incuestionable, y a la búsqueda de una sola respuesta correcta. Saturan en él las subescalas "El conocimiento es cierto" y "Se debe buscar respuestas simples".

Factor 4 Aprendizaje rápido: Este factor se compone de las subescalas "El aprendizaje es rápido" y "El aprendizaje ocurre en el primer intento". El contenido semántico de los ítems que componen ambas subescalas refiere al tiempo que le toma a una persona el aprender algo.

Factor 5 Habilidad fija: Este factor se compone de las subescalas "No se puede aprender a aprender" y "Éxito no relacionado al esfuerzo". El contenido semántico de los ítems que componen ambas subescalas refiere a la dimensión teórica propuesta por la autora denominada habilidad innata o habilidad fija.

Al validarse la estructura factorial del instrumento, se efectuaron análisis estadísticos con las cinco dimensiones. Los resultados mostraron un perfil medio que se inclina ligeramente hacia el polo de la sofisticación en las creencias epistemológicas de la muestra total de investigación (ver tabla 4 y figura 1).

Tabla 4. Medias y desviaciones típicas de los puntajes obtenidos en las cinco dimensiones

\begin{tabular}{lcc}
\hline & Media & Desv. Típ. \\
\hline Conocimiento simple & 2.61 & .27 \\
Conocimiento cierto & 2.45 & .38 \\
Autoridad omnisciente & 2.76 & .32 \\
Habilidad fija & 2.41 & .32 \\
Aprendizaje rápido & 2.24 & .35 \\
\hline
\end{tabular}

Fuente: Elaboración propia con información proveniente de los análisis estadísticos efectuados en el presente estudio. 
Figura 1. Puntuaciones promedio obtenidas para las cinco dimensiones en la muestra total

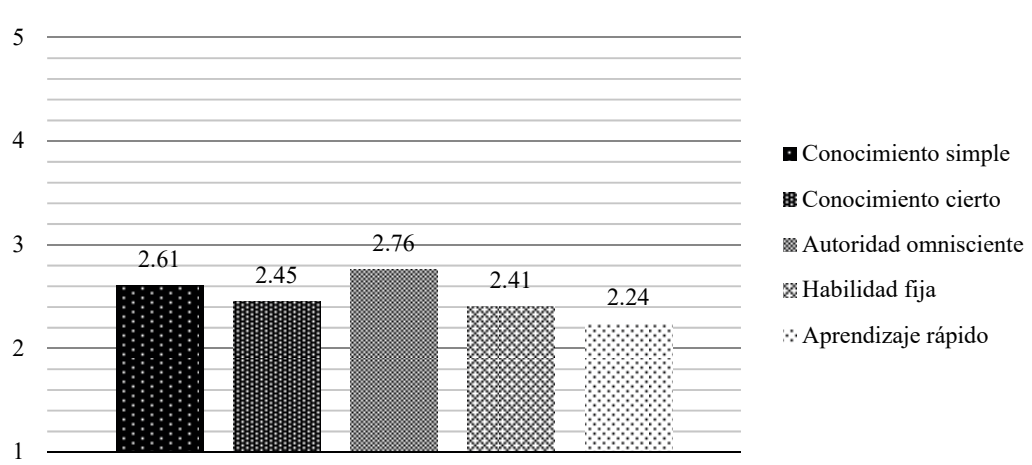

Fuente: Elaboración propia con información proveniente de los análisis estadísticos efectuados en el presente estudio.

\subsection{Contrastes estadísticos de las hipótesis de investigación}

Los contrastes de hipótesis se realizaron con pruebas no paramétricas ya que la prueba de Kolmogorov-Smirnov rechazó la normalidad de 4 de las dimensiones, ajustándose solo las puntuaciones de la dimensión conocimiento cierto a la curva normal. Dicha variable se trató a posteriori como una variable no paramétrica por la decisión de mantener el mismo tipo de pruebas en todas las dimensiones.

\subsubsection{Creencias epistemológicas y momento de formación en los estudios}

Para observar las diferencias en creencias epistemológicas que pudieran ocurrir por acción del momento de formación, se decidió comparar estudiantes de primer y cuarto año de estudios que tuvieran la misma edad. La mayoría de estudiantes de cuarto año $(\mathrm{N}=51)$ reportó tener entre 21 y 22 años de edad, por lo que se seleccionó estudiantes de primer año que se encontraran en el mismo rango etario $(\mathrm{N}=49)$. Los resultados mostraron que existen diferencias significativas, aunque de intensidad pequeña, entre los estudiantes debido al momento de formación (ver tabla 5). El alumnado de primer ańo tiene puntajes significativamente mayores que el alumnado de cuarto año en las dimensiones conocimiento simple ( $p=.01$, con un tamaño del efecto moderado-bajo $\left.r^{1}=-.246\right)$, conocimiento cierto $(p=.00$, con un tamańo del

1 Para la determinación del tamaño de efecto, al tratarse de contrastes no paramétricos con la prueba U de Mann Whitney, lo recomendable, según Fritz, Morris y Richler (2012) es utilizar la r de Rosenthal. 
efecto moderado $r=-.278)$ y habilidad fija $(p=.03$, con un tamaño del efecto moderado-bajo $r=-.208$ ).

Tabla 5. Comparación de medias para los factores del CCE en función del año de estudios

\begin{tabular}{|c|c|c|c|c|}
\hline & \multicolumn{2}{|c|}{ Medias } & \multirow{2}{*}{$\begin{array}{c}\text { U de } \\
\text { Mann-Whitney }\end{array}$} & \multirow{2}{*}{$\begin{array}{l}\text { Sig. Asintót. } \\
\text { (bilateral) }\end{array}$} \\
\hline & $1 \mathrm{r}$ año & $4^{\circ}$ año & & \\
\hline Conocimiento simple & 2.67 & 2.54 & 893.00 & $.01^{*}$ \\
\hline Conocimiento cierto & 2.55 & 2.38 & 846.50 & $.00^{* *}$ \\
\hline Autoridad omnisciente & 2.76 & 2.79 & 1188.50 & .67 \\
\hline Habilidad fija & 2.50 & 2.34 & 948.50 & $.03^{*}$ \\
\hline Aprendizaje rápido & 2.28 & 2.22 & 1179.50 & .62 \\
\hline
\end{tabular}

${ }^{*} p<.05^{* *} p<.01$

Fuente: Elaboración propia con información proveniente de los análisis estadísticos efectuados en el presente estudio.

Por su parte, al analizar diferencias en función del año de estudios por sexo, se encontró que entre las mujeres de primer y cuarto ańo existen diferencias en sus creencias respecto al conocimiento simple $(p=.01)$ y conocimiento cierto $(p=.00)$. Es decir, las estudiantes mujeres de primer año tienden a mostrar mayor ingenuidad respecto a la estructura del conocimiento $\left(M_{\text {simple }}=2.67\right.$, $n=45)$ y certeza del conocimiento $\left(M_{\text {certeza }}=2.57, n=45\right)$, en comparación a las estudiantes mujeres de cuarto ańo $\left(M_{\text {simple }}=2.54\right.$ y $\left.M_{\text {certeza }}=2.38, n=50\right)$. En las tres dimensiones restantes no se encontraron diferencias entre las mujeres.

$\mathrm{El}$ análisis entre hombres de primer y cuarto año no arrojó diferencias significativas en sus creencias epistemológicas.

\subsubsection{Creencias epistemológicas y sexo}

$\mathrm{Al}$ efectuar el contraste de las variables por sexo (ver tabla 6 ), se observó que la única diferencia significativa se dio en la dimensión habilidad fija ( $p=.02$, con un tamańo del efecto bajo $r=-.149)$. La media en las estudiantes mujeres $(M=2.40, n=204)$ fue ligeramente menor en este factor en comparación a la media en los hombres $(M=2.57, n=25)$, por lo que se puede decir que las estudiantes mujeres del presente estudio presentan creencias más sofisticadas en cuanto a la habilidad para aprender, en comparación a sus compañeros varones. 
Tabla 6. Comparación de medias para los factores del CCE en función del sexo

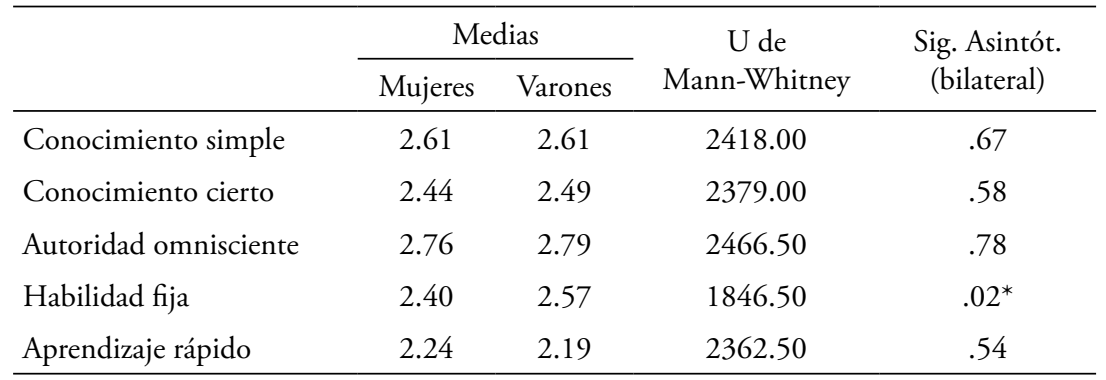

${ }^{*} p<.05$

Fuente: Elaboración propia con información proveniente de los análisis estadísticos efectuados en el presente estudio.

\subsubsection{Creencias epistemológicas y edad}

Para contrastar la hipótesis se efectuaron análisis de correlación de Spearman (ver tabla 7) entre las cinco dimensiones de las creencias epistemológicas y la variable de edad. Sin embargo, no se encontró relaciones significativas entre la edad de los estudiantes y las creencias.

Tabla 7. Correlaciones entre las creencias epistemológicas y la variable de edad

\begin{tabular}{|c|c|c|c|c|c|c|}
\hline & & $\begin{array}{c}\text { Conocimiento } \\
\text { simple }\end{array}$ & $\begin{array}{c}\text { Conocimiento } \\
\text { cierto }\end{array}$ & $\begin{array}{c}\text { Autoridad } \\
\text { omnisciente }\end{array}$ & $\begin{array}{l}\text { Habilidad } \\
\text { fija }\end{array}$ & $\begin{array}{l}\text { Aprendizaje } \\
\text { rápido }\end{array}$ \\
\hline \multirow[t]{2}{*}{ Edad } & $\begin{array}{l}\text { Coefic. de } \\
\text { correlación }\end{array}$ & -.01 & -.05 & -.01 & -.01 & .09 \\
\hline & $\begin{array}{l}\text { Sig. } \\
\text { (bilateral) }\end{array}$ & .80 & .44 & .88 & .80 & .14 \\
\hline
\end{tabular}

Fuente: Elaboración propia con información proveniente de los análisis estadísticos efectuados en el presente estudio.

\section{Discusión DE RESULTADOS}

Uno de los principales objetivos buscó averiguar si podía encontrarse evidencia que valide las cinco dimensiones teóricas que componen según Schommer (1990) las creencias epistemológicas. Por los resultados obtenidos en el análisis factorial exploratorio de las respuestas al Cuestionario de Creencias Epistemológicas aplicado a la muestra de estudiantes de pedagogía de la Universidad de Barcelona, se concluyó que la estructura factorial hallada 
y su interpretación son congruentes con la propuesta teórica de la autora (Schommer, 1990, 1993). Los resultados son importantes puesto que es la primera vez que una investigación, de carácter exploratorio, logra validar la totalidad del modelo epistemológico de Schommer. Anteriores investigaciones con muestras españolas (De Juanas, Navarro \& González, 2011; Rodríguez, 2005) han validado solo cuatro de las cinco dimensiones.

Los resultados revelaron que el perfil medio de las creencias de la muestra total de estudiantes se acercó ligeramente al polo de la sofisticación. No obstante, de las cinco dimensiones, autoridad omnisciente fue la más ingenua. Especialmente llamó la atención que el alumnado de cuarto año no mostrara diferencias significativas en dicha dimensión respecto al alumnado de primer ańo, lo cual sugiere la reflexión acerca de cómo desarrollar en los estudiantes posturas de mayor autonomía en la construcción y generación del conocimiento.

En cuanto al segundo objetivo de investigación, se observaron variaciones significativas en las creencias de los estudiantes de primer y cuarto año, solo respecto al conocimiento simple, conocimiento cierto y habilidad fija. Contar con más años de formación se desplegó en el presente estudio como un factor asociado a que dichas creencias varíen hacia la sofisticación. Estos resultados concuerdan en parte con los obtenidos por Schommer (1998), quien encontró que la experiencia educativa es un predictor de las creencias referidas a la estructura y certeza del conocimiento.

De igual manera destacó la existencia de diferencias entre las mujeres de primer y cuarto ańo en las dimensiones conocimiento simple y conocimiento cierto. Resultados que confirman nuevamente los obtenidos por Schommer (1993, 1998) quien encontró que la educación predice las creencias acerca de la estructura y estabilidad del conocimiento. Entre los hombres de primer y cuarto año, por su parte, no se encontraron diferencias significativas, lo cual puede deberse a que la muestra de varones en el presente estudio fue muy pequeña. Lamentablemente en las carreras relacionadas con la educación o pedagogía se cuenta con una cuota masculina muy limitada, por lo que investigaciones que integren perspectivas cuantitativas y cualitativas podrían ser de mucha utilidad para conocer de una manera más profunda las concepciones de estos sujetos acerca de la naturaleza del conocimiento y el aprendizaje.

Otro de los objetivos del estudio fue identificar si existen diferencias en las creencias epistemológicas debido al sexo, encontrándose que las mujeres muestran creencias más sofisticadas que los varones en la dimensión habilidad fija. 
En las otras cuatro dimensiones no se encontraron diferencias. Ya Schommer (1993) había señalado anteriormente que las mujeres suelen tener una ventaja epistemológica en algunas dimensiones, por lo que este hallazgo concuerda parcialmente con los resultados empíricos previos (De Juanas \& Beltrán, 2012; Hofer, 2000; Schommer, 1993), en el sentido de que es frecuente encontrar en las mujeres la creencia de que la habilidad para aprender puede mejorarse a través de la experiencia y el tiempo dedicado al aprendizaje.

Por último, no se encontró relación entre las creencias epistemológicas y la edad. Esto puede deberse a que como bien manifiesta Schommer (1998), la edad es una medida global del desarrollo asociada a la madurez y experiencias de vida, por lo que las experiencias de vida específicas pueden mostrar una relación más fuerte con las creencias epistemológicas que la variable edad.

\section{RECOMENDACIONES}

A pesar del hallazgo de los cinco factores, algunas subescalas saturaron de un modo considerable en más de un factor. Ello podría deberse a que las subescalas y los ítems que las componen contienen un alto grado de dependencia, por lo que es recomendable que posteriores investigaciones, especialmente transculturales (Bernardo, 2008; Braten \& Strømsø, 2005), profundicen en la validez de constructo y en la consistencia interna del cuestionario.

Las diferencias halladas debido al momento de formación y al sexo, aunque son significativas estadísticamente, tienen un tamaño del efecto bajo o moderado-bajo, por lo que los resultados deben ser tomados con cautela y solo pueden ser generalizados a estudiantes del contexto cultural específico de este estudio y de condiciones similares a las características de la muestra. Aún estos resultados son importantes puesto que es la primera vez que un estudio reporta las creencias epistemológicas de estudiantes universitarios en el contexto de Cataluña. Asimismo, es también la primera vez que un análisis factorial al Cuestionario de Creencias Epistemológicas de Schommer estructura las cinco dimensiones teóricas. Investigadores ya han comentado que las creencias pueden ser dependientes del contexto y valores culturales, por lo que estudios futuros podrían profundizar y confirmar la multidimensionalidad de las creencias epistemológicas de estudiantes de otros contextos y profesiones. 


\section{REFERENCIAS BIBLIOGRÁFICAS}

Bernardo, A. (2008). Exploring epistemological beliefs of bilingual filipino preservice teachers in the filipino and english languages. The Journal of Psychology, 142(2), 193-208. https://doi.org/10.3200/JRLP.142.2.193-208

Braten, I. \& Strømsø, H. I. (2005). The relationship between epistemological beliefs, implicit theories of intelligence, and self-regulated learning among Norwegian postsecondary students. British Journal of Educational Psychology, 75, 539-565. https://doi.org/10.1348/000709905X25067

Buehl, M. \& Alexander, A. (2006). Examining the dual nature of epistemological beliefs. International Journal of Educational Research, 45, 28-42. https:// doi.org/10.1016/j.ijer.2006.08.007

Cho, M. \& Lankford, D. M. (2011). Exploring the Relationships among Epistemological Beliefs, Nature of Science, and Conceptual Change in the Learning of Evolutionary Theory. Evolution: Education and Outreach, 4, 313-322. https://doi.org/10.1007/s12052-011-0324-7

Coşkun, M. \& Grainger, P. (2014). The effect of epistemological beliefs on teaching-learning conceptions of pre-service teachers of religion. Global Journal of Teacher Education, 2(3), 176-184.

De Juanas, A. \& Beltrán, J. (2012). Creencias epistemológicas de los estudiantes de Pedagogía y Ciencias de la Educación. Revista de Psicodidáctica, 17(1), 179-198.

De Juanas, A., Navarro, E. \& González, A. (2011). Estudio confirmatorio de la estructura factorial del Cuestionario de Creencias Epistemológicas con una muestra de estudiantes universitarios de la Universidad Complutense de Madrid. VI Congreso Internacional de Psicología y Educación. III Congreso Nacional de Psicología de la Educación. Valladolid: Universidad de Valladolid.

Di Matteo, M. (2007). Creencias epistemológicas acerca de la naturaleza del conocimiento en estudiantes de ciencias de la educación y profesorados universitarios. IV Jornadas de Jóvenes Investigadores. Buenos Aires: Instituto de Investigaciones Gino Germani, Facultad de Ciencias Sociales, Universidad de Buenos Aires.

Duell, O. K. \& Schommer-Aikins, M. (2001). Measures of people's beliefs about knowledge and learning. Educational Psychology Review, 13(4), 419-449. https://doi.org/10.1023/A:1011969931594

Fritz, C. O., Morris, P. E. \& Richler, J. J. (2012). Effect Size Estimates: Current Use, Calculations, and Interpretation. Journal of Experimental Psychology: General, 141(1), 2-18. https://doi.org/10.1037/a0024338 
García, M. \& Sebastián, C. (2011). Creencias epistemológicas de los estudiantes de pedagogía en educación parvularia, básica y media: ¿Diferencias en la formación inicial docente? Psykhé, 20(1), 29-43. https://doi.org/10.4067/ S0718-22282011000100003

Gill, M., Ashton, P. \& Algina, J. (2004). Changing preservice teachers' epistemological beliefs about teaching and learning in mathematics: an intervention study. Contemporary Educational Psychology, 29, 164-185. https://doi. org/10.1016/j.cedpsych.2004.01.003

Han, H. \& Jeong, C. (2014). Improving Epistemological Beliefs and Moral Judgment through an STS-Based Science Ethics Education Program. Science and Engineering Ethics, 20, 197-220. https://doi.org/10.1007/ s1 1948-013-9429-4

Hofer, B. K. (2000). Dimensionality and disciplinary differences in personal epistemology. Contemporary Educational Psychology, 25, 378-405. https://doi. org/10.1006/ceps.1999.1026

Hofer, B. K. (2001). Personal Epistemology Research: Implications for Learning and Teaching. Journal of Educational Psychology Review, 13(4), 354-383. https://doi.org/10.1023/A:1011965830686

Hofer, B. K. (2004). Exploring the dimensions of personal epistemology in differing classroom contexts: Student interpretations during the first year of college. Contemporary Educational Psychology, 29, 129-163. https://doi. org/10.1016/j.cedpsych.2004.01.002

Hofer, B. K. \& Pintrich, P. R. (1997). The development of epistemological theories: beliefs about knowledge and knowing and their relation to learning. Review of Educational Research, 67, 88-140. https://doi. org/10.3102/00346543067001088

Hofer, B. K. \& Pintrich, P. R. (2002). Personal epistemology: The psychology of beliefs about knowledge and knowing. Mahwah, NJ: Lawrence Erlbaum Associates.

Jehng, J., Johnson, S. \& Anderson, R. (1993). Schooling and student's epistemological beliefs about learning. Contemporary Educational Psychology, 18, 23-35. https://doi.org/10.1006/ceps.1993.1004

Kember, D., Hong, C., Yau, V. \& Ho, A. (2014). Is it the teaching or the discipline? Influences of disciplinary epistemology and pedagogy on students adapting study behaviour and epistemological beliefs. European Journal of Higher Education, 4(4), 348-362. https://doi.org/10.1080/21568235.20 14.930794 
Martínez, R., Montero, Y. \& Pedrosa, M. (2009). Una aproximación a las creencias epistemológicas y pedagógicas de los profesores y a su valoración de internet. Revista Iberoamericana de Educación, 51(1), 1-15.

Mason, L. \& Boscolo, P. (2004). Role of epistemological understanding and interest in interpreting a controversy and in topic-specific belief change. Contemporary Educational Psychology, 29, 103-128. https://doi. org/10.1016/j.cedpsych.2004.01.001

Mohamed, M. \& El-Habbal, M. (2013). The relationship between epistemic beliefs and academic performance: Are better students always more mature? Journal of Educational and Developmental Psychology, 3(1), 158172. https://doi.org/10.5539/jedp.v3n1p158

Müller, S., Rebmann, K. \& Liebsch, E. (2008). Las creencias de los formadores acerca del conocimiento y el aprendizaje: un estudio piloto. Revista Europea de Formación Profesional, 45, 99-119.

Ozkal, K., Tekkaya, C., Sungur, S., Cakiroglu, J. \& Cakiroglu, E. (2011). Elementary Students' Scientific Epistemological Beliefs in Relation to Socio-Economic Status and Gender. Journal of Science Teacher Education, 22, 115-127. https://doi.org/10.1007/s10972-010-9197-9

Pecharromán, I. \& Pozo, J. I. (2010). ¿Cómo sé que es bueno? Creencias epistemológicas en el dominio moral. Revista de Educación, 353, 387-414.

Perry, W. (1968). Patterns of development in thought and values of students in a liberal arts college: a validation of a scheme. Cambridge, MA: Bureau of Study Counsel, Harvard University.

Rodríguez, L. (2005). Análisis de las creencias epistemológicas, concepciones y enfoques de aprendizaje de los futuros profesores. Tesis doctoral no publicada, Universidad de Granada, Granada, España.

Schommer, M. (1990). Effects of beliefs about the nature of knowledge on comprehension. Journal of Educational Psychology, 82, 498-504. https://doi. org/10.1037/0022-0663.82.3.498

Schommer, M. (1993). Epistemological development and academic performance among secondary students. Journal of Educational Psychology, 85(3), 406411. https://doi.org/10.1037/0022-0663.85.3.406

Schommer, M. (1998). The influence of age and education on epistemological beliefs. British Journal of Educational Psychology, 68, 551-562. https://doi. org/10.1111/j.2044-8279.1998.tb01311.x

Schommer-Aikins, M. (2004). Explaining the Epistemological Beliefs System: Introducing the Embedded Systemic Model and Coordinated Research Approach. Educational Psychologist, 39(1), 19-29. https://doi.org/10.1207/ s15326985ep3901_3 
Schommer-Aikins, M., Beuchat-Reichardt, M. \& Hernández-Pina, F. (2012). Creencias epistemológicas y de aprendizaje en la formación inicial de profesores. Anales de Psicología, 28(2), 465-474. https://doi.org/10.6018/ analesps

Schommer-Aikins \& Duell, O. K. (2013). Domain specific and general epistemological beliefs. Their effects on mathematics. Revista de Investigación Educativa, 31(2), 317-330. https://doi.org/10.6018/rie.31.2.170911

Schommer-Aikins, M. \& Easter, M. (2008). Epistemological Beliefs' Contributions to Study Strategies of Asian Americans and European Americans. Journal of Educational Psychology, 100(4), 920-929. https:// doi.org/10.1037/0022-0663.100.4.920

Topcu, M. S. (2013). Preservice teachers' epistemological beliefs in Physics, Chemistry, and Biology: a mixed study. International Journal of Science and Mathematics Education, 11(2), 433-458. https://doi.org/10.1007/ s10763-012-9345-0

Trautwein, U. \& Lüdtke, O. (2007). Predicting global and topic-specific certainty beliefs: Domain-specificity and the role of the academic environment. British Journal of Educational Psychology, 77, 907-934. https://doi. org/10.1348/000709906X169012

Wang, X., Zhang, Z., Zhang, X. \& Hou, D. (2013). Validation of the chinese version of the Epistemic Beliefs Inventory using confirmatory factor analysis. International Education Studies, 6(8), 98-111. https://doi.org/10.5539/ies. v6n8p98 